\title{
REAL ZEROS OF RANDOM ALGEBRAIC POLYNOMIALS WITH BINOMIAL ELEMENTS
}

\author{
A. NEZAKATI AND K. FARAHMAND
}

Received 26 August 2005; Revised 26 September 2005; Accepted 26 September 2005

This paper provides an asymptotic estimate for the expected number of real zeros of a random algebraic polynomial $a_{0}+a_{1} x+a_{2} x^{2}+\cdots+a_{n-1} x^{n-1}$. The coefficients $a_{j}(j=$ $0,1,2, \ldots, n-1)$ are assumed to be independent normal random variables with mean zero. For integers $m$ and $k=O(\log n)^{2}$ the variances of the coefficients are assumed to have nonidentical value $\operatorname{var}\left(a_{j}\right)=\left(\begin{array}{c}k-1 \\ j-i k\end{array}\right)$, where $n=k \cdot m$ and $i=0,1,2, \ldots, m-1$. Previous results are mainly for identically distributed coefficients or when $\operatorname{var}\left(a_{j}\right)=\left(\begin{array}{c}n \\ j\end{array}\right)$. We show that the latter is a special case of our general theorem.

Copyright (c) 2006 A. Nezakati and K. Farahmand. This is an open access article distributed under the Creative Commons Attribution License, which permits unrestricted use, distribution, and reproduction in any medium, provided the original work is properly cited.

\section{Introduction}

Let $(\Omega, \operatorname{Pr}, \mathscr{A})$ be a fixed probability space and

$$
P_{n}(x)=\sum_{j=0}^{n-1} a_{j} x^{j}
$$

where for $\omega \in \Omega, a_{j}(\omega) \equiv a_{j}, j=0,1,2, \ldots, n-1$, is a sequence of random variables defined on $\Omega$. Denote $N_{n}(a, b)$ as the number of real zeros of $P_{n}(x)$ in the interval $(a, b)$. One way to determine the mathematical behavior of $P_{n}(x)$ is by looking at $\operatorname{EN}_{n}(a, b)$, its expected number of real zeros. This has been achieved for many forms of $P_{n}(x)$ and varieties of assumptions for the distributions of $a_{j}$ 's. However, the majority of results assume identical distributions for the coefficients, albeit, $E N_{n}$ is now known for most classes of distributions, see for example Ibragimov and Maslova [9] or Farahmand [7] and its references. Recently, in an interesting work Edelman and Kostlan [6] introduced a case of nonidentical coefficients in which $\operatorname{var}\left(a_{j}\right)=\left(\begin{array}{c}n \\ j\end{array}\right), j=0,1,2, \ldots, n-1$. Indeed, the above 
case is also motivated by several physical applications which are studied, for example, in Ramponi [11], Bleher and Di [2, 3], Bogomolny et al. [4, 5], and Aldous and Fyodorov [1]. There have been further works to study the mathematical behavior of $E N_{n}$ of which the most recent is Farahmand and Nezakati [8].

The special feature of choosing the above assumptions for the $\operatorname{var}\left(a_{j}\right)$ 's, indeed beside physical applications mentioned above, is the significant increase to $E N_{n}(-\infty, \infty)$. In fact this expected number increases from $(2 / \pi) \log n$ for the case of identical normal standard to $\sqrt{n}$ for the case when $a_{j}(\omega)$ is normal with mean zero and variance $\left(\begin{array}{c}n \\ j\end{array}\right)$. This assumption for the variances of the coefficients introduces a new class of polynomials in which there are more zeros than algebraic polynomials with identical variances and less than random trigonometric polynomials defined as $\sum_{j=0}^{n-1} a_{j} \cos j \theta$. The expected number of real zeros of the latter polynomial is $\operatorname{EN}_{n}(0,2 \pi) \sim n / \sqrt{3}$. Indeed, these results show that the oscillatory behavior of the above random polynomials has the least number of oscillations for the algebraic with identical coefficients case and most for the trigonometric case. Our case of random algebraic polynomial with nonidentical coefficients has a number of oscillations between these two extreme cases. Therefore, it is natural to ask whether or not for other cases of nonidentical variances with binomial elements, the latter increases in $E N_{n}$ remain stainable. To this end we prove the following theorem.

Theorem 1.1. For random algebraic polynomial $P_{n}(x)$, let $n$ be separated into two multipliers such that $n=k \cdot m$, where $k=f(n)$ is an integer and increasing function of $n$, such that $f(n)=O(\log n)^{2}$. The random variables $a_{j}, j=0,1,2, \ldots, n-1$ are normally distributed with means zero and $\operatorname{var}\left(a_{j}\right)=\left(\begin{array}{c}k-1 \\ j-i k\end{array}\right), j=i k, i k+1, \ldots,(i+1) k-1, i=0,1, \ldots, m-1$. Then for sufficiently large $n$, the expected number of real zeros of $P_{n}(x)$ is

$$
E N_{n}(-\infty, \infty) \sim \sqrt{k-1}
$$

\section{Proof of theorem}

For proof of the theorem we use an approach based on Kac's [10] or Rice's [12] results. In this case for

$$
\begin{gathered}
A^{2}=\operatorname{var}\left(P_{n}(x)\right), \quad B^{2}=\operatorname{var}\left(P_{n}^{\prime}(x)\right), \\
C=\operatorname{cov}\left(P_{n}(x), P_{n}^{\prime}(x)\right), \quad \Delta^{2}=A^{2} B^{2}-C^{2},
\end{gathered}
$$

the expected number of real zeros is given by the Kac-Rice formula as

$$
\operatorname{EN}_{n}(a, b)=\frac{1}{\pi} \int_{a}^{b} \frac{\Delta}{A^{2}} d x
$$

In order to use (2.2) to obtain $E N_{n}(-\infty, \infty)$ we note that changing $x$ to $1 / x$ and $x$ to $-x$ leaves the distribution of the coefficients of $P_{n}(x)$ in (1.1) invariant. Hence the expected number of real zeros in the interval $(0,1)$ is asymptotically the same as in $(1, \infty),(-1,0)$, and $(-\infty,-1)$. Therefore it suffices to give the result for $\operatorname{EN}_{n}(0,1)$ only. To this end, we present our calculations for any integer $k$. From the assumptions of Theorem 1.1 for the 
distributions of the coefficients of $P_{n}(x)$, from (2.1) we can easily show that

$$
A^{2}=\sum_{j=0}^{n-1} \operatorname{var}\left(a_{j}\right) x^{2 j}, \quad B^{2}=\sum_{j=0}^{n-1} \operatorname{var}\left(a_{j}\right) j^{2} x^{2 j-2}, \quad C=\sum_{j=0}^{n-1} \operatorname{var}\left(a_{j}\right) j x^{2 j-1} .
$$

Now similar to the method of Sambandham [13], let

$$
H(x, y)=\sum_{j=0}^{n-1} \operatorname{var}\left(a_{j}\right) x^{j} y^{j}
$$

then we can obtain

$$
\begin{aligned}
H(x, y)= & \left(\begin{array}{c}
k-1 \\
0
\end{array}\right)+\left(\begin{array}{c}
k-1 \\
1
\end{array}\right) x y+\cdots+\left(\begin{array}{l}
k-1 \\
k-1
\end{array}\right)(x y)^{k-1} \\
& +(x y)^{k}\left[\left(\begin{array}{c}
k-1 \\
0
\end{array}\right)+\left(\begin{array}{c}
k-1 \\
1
\end{array}\right) x y+\cdots+\left(\begin{array}{l}
k-1 \\
k-1
\end{array}\right)(x y)^{k-1}\right]+\cdots \\
& +(x y)^{(m-1) k}\left[\left(\begin{array}{c}
k-1 \\
0
\end{array}\right)+\left(\begin{array}{c}
k-1 \\
1
\end{array}\right) x y+\cdots+\left(\begin{array}{l}
k-1 \\
k-1
\end{array}\right)(x y)^{k-1}\right] \\
= & \left(1+x^{2}\right)^{k-1}\left[1+(x y)^{k}+(x y)^{2 k}+\cdots+(x y)^{(m-1) k}\right] \\
= & \frac{1-x^{n} y^{n}}{1-x^{k} y^{k}}(1+x y)^{k-1} .
\end{aligned}
$$

Therefore from (2.3)-(2.4) we can easily see that

$$
A^{2}=H(x, x), \quad B^{2}=\left[\frac{\partial^{2} H(x, y)}{\partial x \partial y}\right]_{y=x}, \quad C=\left[\frac{\partial H(x, y)}{\partial x}\right]_{y=x} .
$$

Therefore it is an easy exercise to obtain the value of $A^{2}, B^{2}$, and $C$ given in (2.3) as

$$
\begin{aligned}
A^{2}= & \frac{1-x^{2 n}}{1-x^{2 k}}\left(1+x^{2}\right)^{k-1}, \\
B^{2}= & {\left[\frac{k^{2} x^{4 k-2}+(n-k)^{2} x^{2 n+4 k-2}+\left(2 n^{2}-2 n k-k^{2}\right) x^{2 n+2 k-2}}{\left(1-x^{2 k}\right)^{3}}\right.} \\
& \left.+\frac{k^{2} x^{2 k-2}-n^{2} x^{2 n-2}}{\left(1-x^{2 k}\right)^{3}}\right]\left(1+x^{2}\right)^{k-1}+2(k-1)\left[\frac{(n-k) x^{2 n+2 k}+k x^{2 k}-n x^{2 n}}{\left(1-x^{2} k\right)^{2}}\right]\left(1+x^{2}\right)^{k-2} \\
& +\left[\frac{(k-1)\left(1+k x^{2}-x^{2}\right)\left(1-x^{2 n}\right)}{1-x^{2 k}}\right]\left(1+x^{2}\right)^{k-3}, \\
C= & {\left[\frac{(n-k) x^{2 n+2 k-1}+k x^{2 k-1}-n x^{2 n-1}}{\left(1-x^{2 k}\right)^{2}}\right]\left(1+x^{2}\right)^{k-1}+\left[\frac{(k-1) x\left(1-x^{2 n}\right)}{1-x^{2 k}}\right]\left(1+x^{2}\right)^{k-2} . }
\end{aligned}
$$


4 Real zeros of random algebraic polynomials

Also from (2.1) and (2.7), for any integer $k$, we obtain

$$
\begin{aligned}
\frac{\Delta^{2}}{A^{4}}= & \frac{k^{2} x^{2 k-2}-n^{2} x^{2 n-2}+2\left(n^{2}-k^{2}\right) x^{2 n+2 k-2}+\left(n^{2}+2 k^{2}-4 n k\right) x^{2 n+4 k-2}}{\left(1-x^{2 k}\right)^{2}\left(1-x^{2 n}\right)^{2}} \\
& +\frac{k^{2} x^{4 n+2 k-2}-2(n-k)^{2} x^{4 n+4 k-2}}{\left(1-x^{2 k}\right)^{2}\left(1-x^{2 n}\right)^{2}}+\frac{k-1}{\left(1+x^{2}\right)^{2}} .
\end{aligned}
$$

In order to continue the proof of Theorem 1.1, we first consider the interval $(0,1-\eta)$, where for

$$
a=1-\frac{\log \log k^{10}}{\log k}, \quad \text { we let } \eta=k^{-a} .
$$

In this interval, for sufficiently large $n$, we can easily show that

$$
x^{k} \leq k^{-10}, \quad x^{n} \leq k^{-10 m} .
$$

Also note $a \rightarrow 1$ as $n \rightarrow \infty$. This is necessary to obtain the result later. Now from (2.8), we have

$$
\frac{\Delta^{2}}{A^{4}} \sim \frac{k-1}{\left(1+x^{2}\right)^{2}}
$$

Therefore from (2.2), we can show that

$$
\operatorname{EN}_{n}(0,1-\eta) \sim \frac{1}{\pi} \int_{0}^{1-\eta} \frac{\sqrt{k-1}}{1+x^{2}} d x=\frac{\sqrt{k-1}}{\pi} \arctan (1-\eta) .
$$

Hence for sufficiently large $n$,

$$
\operatorname{EN}_{n}(0,1-\eta) \sim \frac{\sqrt{k-1}}{4} .
$$

Now we assume $1-\eta \leq x \leq 1-\delta$, where for

$$
b=1-\frac{\log \log k^{1 / 2}}{\log k}, \quad \text { we let } \delta=k^{-b} .
$$

In this interval, for sufficiently large $n$, we can easily show that

$$
x^{2 k} \leq k^{-1}, \quad x^{2 n} \leq k^{-m} .
$$

Now from (2.8), we have

$$
\frac{\Delta}{A^{2}} \sim \sqrt{k^{2} x^{2 k-2}+\frac{k-1}{\left(1+x^{2}\right)^{2}}} \leq B \sqrt{k}
$$

where $B$ is constant. Therefore, from (2.2), we can show that

$$
E_{n}(1-\eta, 1-\delta)=O\left(k^{-1 / 2} \log k\right)
$$


When $1-\delta \leq x \leq 1-\epsilon$, where for

$$
c=1-\frac{\log \log n^{10}}{\log n}, \quad \text { we let } \epsilon=n^{-c} .
$$

In this interval, for sufficiently large $n$, we can easily show that

$$
k x^{2 k} \geq 1, \quad x^{n} \leq n^{-10} .
$$

Now from (2.8), (2.14), and (2.18), we have

$$
\frac{\Delta}{A^{2}} \sim \frac{k x^{k-1}}{1-x^{2 k}} \sqrt{1+\frac{(k-1)\left(1-x^{2 k}\right)^{2}}{k^{2} x^{2 k-2}\left(1+x^{2}\right)^{2}}} \leq C\left[\frac{k x^{k-1}}{1+x^{k}}+\frac{k x^{k-1}}{1-x^{k}}\right],
$$

where $C$ is constant. Therefore, from (2.2), we can show that

$$
E N_{n}(1-\delta, 1-\epsilon) \leq \frac{C}{\pi} \int_{1-\delta}^{1-\epsilon}\left[\frac{k x^{k-1}}{1+x^{k}}+\frac{k x^{k-1}}{1-x^{k}}\right] d x=\frac{C}{\pi}\left[\log \frac{1+x^{k}}{1-x^{k}}\right]_{1-\delta}^{1-\epsilon}
$$

Therefore, for sufficiently large $n$,

$$
E N_{n}(1-\delta, 1-\epsilon)=O(\log n) .
$$

Finally, let $1-\epsilon \leq x \leq 1$, we know that always

$$
\frac{\Delta}{A^{2}} \leq n
$$

Therefore, from (2.2) and (2.18), and for sufficiently large $n$, we also have,

$$
\operatorname{EN}_{n}(1-\epsilon, 1)=O(\log n)
$$

Hence from (2.13), (2.17), (2.22), and (2.24), we have the proof of Theorem 1.1.

Example 2.1. In Theorem 1.1, if $k=n$, then our Theorem 1.1 result is similar to that obtained by Edelman and Kostlan [6].

Example 2.2. In random polynomials $P_{n}(x)=\sum_{j=0}^{n-1} a_{j} x^{j}$, where $n \in\left\{i^{2} ; i=1,2, \ldots\right\}$, if $k=\sqrt{n}$ and conditions of Theorem 1.1 hold, then for sufficiently large $n$,

$$
E N_{n}(-\infty, \infty)=\sqrt{\sqrt{n}-1}
$$

Example 2.3. In random polynomials $P_{n}(x)=\sum_{j=0}^{n-1} a_{j} x^{j}, n \in\left\{i^{3} ; i=1,2, \ldots\right\}$, if $k=n^{1 / 3}$ and conditions of Theorem 1.1 hold, then for sufficiently large $n$,

$$
\operatorname{EN}_{n}(-\infty, \infty)=\sqrt{n^{1 / 3}-1}
$$

and, if $k=n^{2 / 3}$, then for sufficiently large $n$,

$$
\operatorname{EN}_{n}(-\infty, \infty)=\sqrt{n^{2 / 3}-1}
$$




\section{Real zeros of random algebraic polynomials}

\section{References}

[1] A. P. Aldous and Y. V. Fyodorov, Real roots of random polynomials: universality close to accumulation points, Journal of Physics A: Mathematical and General 37 (2004), no. 4, 1231-1239.

[2] P. Bleher and X. Di, Correlations between zeros of a random polynomial, Journal of Statistical Physics 88 (1997), no. 1-2, 269-305.

[3] __ Correlations between zeros of non-Gaussian random polynomials, International Mathematics Research Notices 2004 (2004), no. 46, 2443-2484.

[4] E. Bogomolny, O. Bohigas, and P. Leboeuf, Distribution of roots of random polynomials, Physical Review Letters 68 (1992), no. 18, 2726-2729.

[5] _ Quantum chaotic dynamics and random polynomials, Journal of Statistical Physics 85 (1996), no. 5-6, 639-679.

[6] A. Edelman and E. Kostlan, How many zeros of a random polynomial are real?, Bulletin of the American Mathematical Society 32 (1995), no. 1, 1-37.

[7] K. Farahmand, Topics in Random Polynomials, Pitman Research Notes in Mathematics Series, vol. 393, Longman, Harlow, 1998.

[8] K. Farahmand and A. Nezakati, Algebraic polynomials with non-identical random coefficients, Proceedings of the American Mathematical Society 133 (2005), no. 1, 275-283.

[9] I. A. Ibragimov and N. B. Maslova, On the expected number of real zeros of random polynomials. II. Coefficients with non-zero means, Theory of Probability and Its Applications 16 (1971), no. 3, 485-493.

[10] M. Kac, On the average number of real roots of a random algebraic equation, Bulletin of the American Mathematical Society 49 (1943), 314-320.

[11] A. Ramponi, A note on the complex roots of complex random polynomials, Statistics \& Probability Letters 44 (1999), no. 2, 181-187.

[12] S. O. Rice, Mathematical analysis of random noise, The Bell System Technical Journal 24 (1945), 46-156, reprinted in: Selected Papers on Noise and Stochastic Processes (N. Wax ed.), Dover, New York, 1954, 133-294.

[13] M. Sambandham, On a random algebraic equation, Journal of the Indian Mathematical Society 41 (1977), no. 1-2, 83-97.

A. Nezakati: Faculty of Mathematics, Shahrood University of Technology, P.O. Box 316-36155, Shahrood, Iran

E-mail address: nezakati@shahrood.ac.ir

K. Farahmand: Department of Mathematics, University of Ulster, Jordanstown Campus, County Antrim BT37 0QB, UK

E-mail address: k.farahmand@ulster.ac.uk 


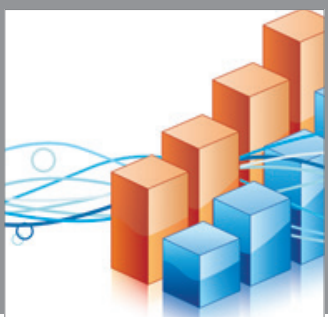

Advances in

Operations Research

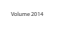

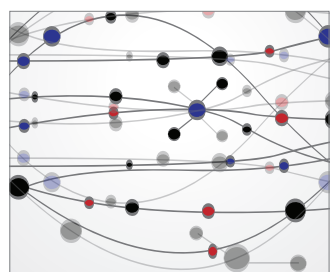

\section{The Scientific} World Journal
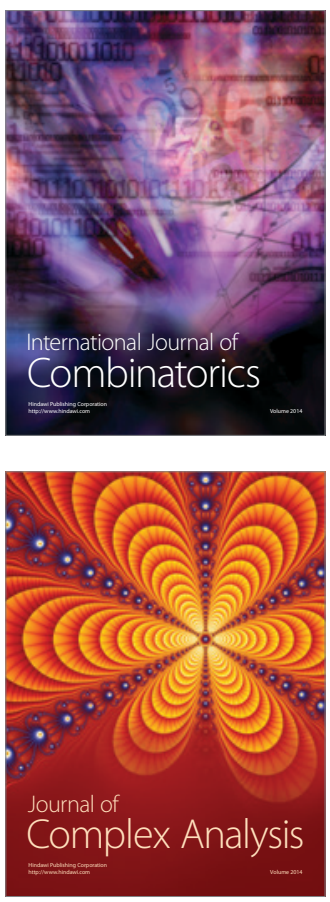

International Journal of

Mathematics and

Mathematical

Sciences
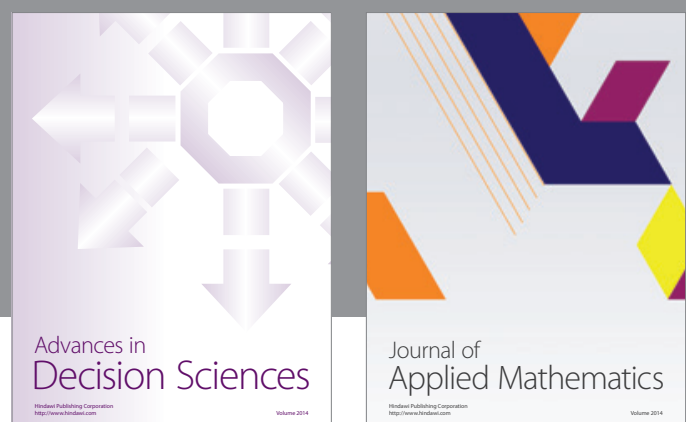

Journal of

Applied Mathematics
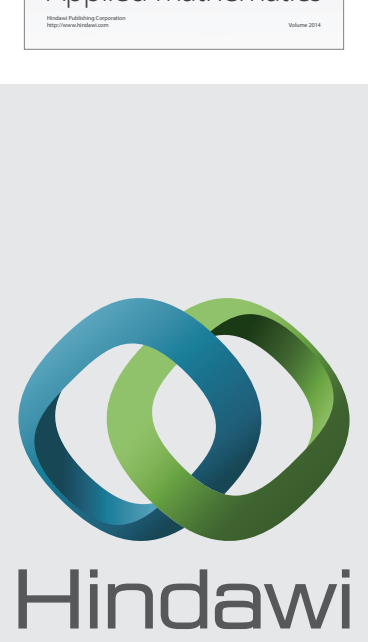

Submit your manuscripts at http://www.hindawi.com
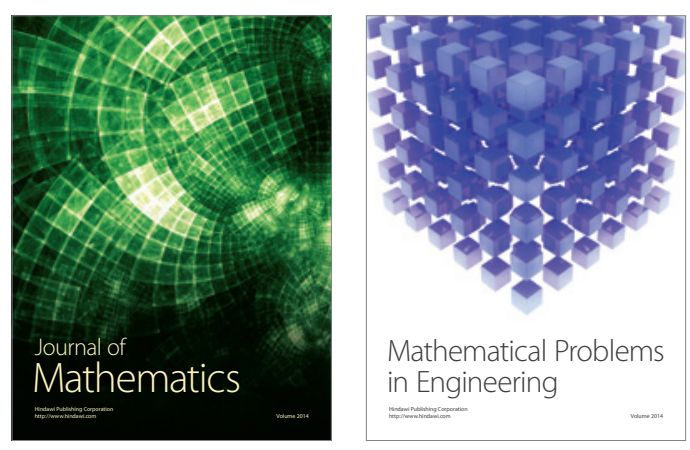

Mathematical Problems in Engineering
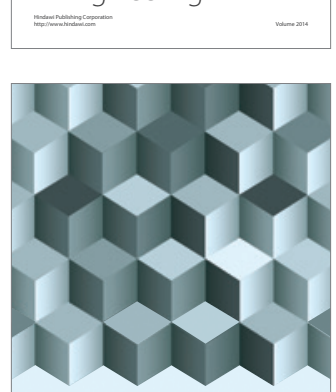

Journal of

Function Spaces
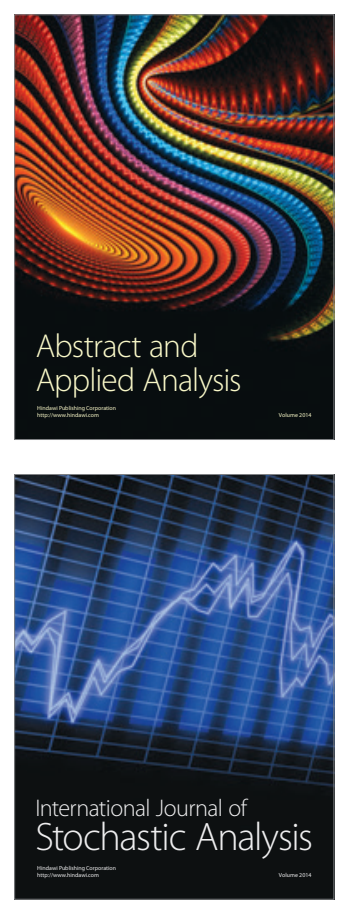

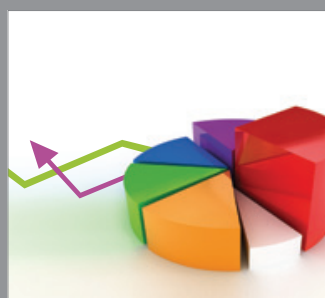

ournal of

Probability and Statistics

Promensencen
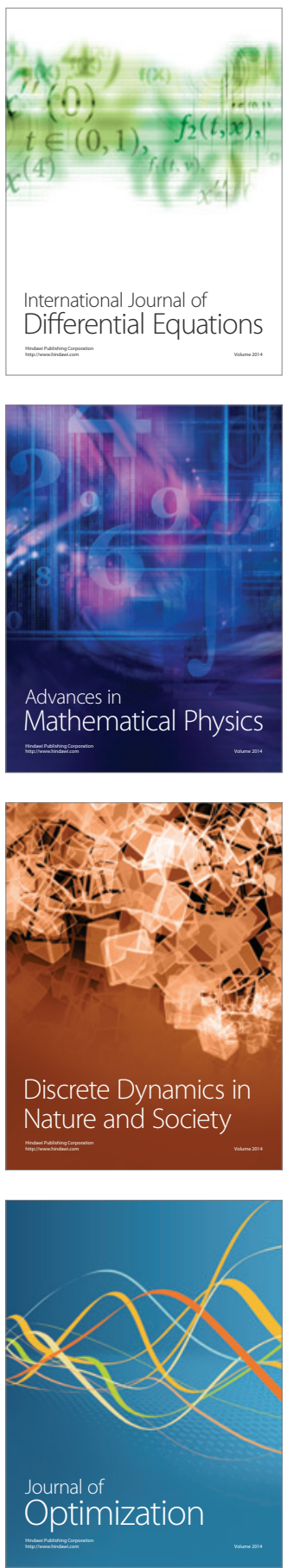